\title{
A NOTE ON LOCAL DENSITIES OF QUADRATIC FORMS
}

\author{
YOSHIYUKI KITAOKA
}

Let $L, M$ be regular quadratic lattices over $Z_{p}$. The local density $\alpha_{p}(L, M)$ is an important invariant in the theory of representation of quadratic forms and they appear naturally in Fourier coefficients of Eisenstein series. In spite of the importance we knew little except the case when either $L=M$ or $\operatorname{rk} L=1$ and $M$ is unimodular. Evaluating them is a laborious task. Recently M. Ozeki gave an formula of local densities $\alpha_{p}(L, M)$, where $\operatorname{rk} L=2, M$ is unimodular and $p \neq 2$, by calculating generalized Gauss sums. Here we give a general induction formula and a simpler proof of his results as an application and interesting relations which are not referred in [4], [5].

For a prime $p$, we denote by $\boldsymbol{Q}_{p}, \boldsymbol{Z}_{p}$ the $p$-adic completion of the rational number field $\boldsymbol{Q}$, the rational integer ring $Z$ respectively.

Let $G=G L_{n}\left(\boldsymbol{Q}_{p}\right) \cap M_{n}\left(\boldsymbol{Z}_{p}\right), U=G L_{n}\left(\boldsymbol{Z}_{p}\right)$. By $H_{n}\left(\boldsymbol{Q}_{p}\right)$ we denote the vector space over $\boldsymbol{Q}$ consisting of all formal finite linear combinations with coefficients in $\boldsymbol{Q}$ of the double cosets $U g U, g \in G$. Let $g, h \in G$ and $U g U=U g_{1} \cup \cdots \cup U g_{s}, U h U=U h_{1} \cup \cdots \cup U h_{t}$ be left cosets decompositions of $U g U, U h U$ respectively. For every $k \in G$, the number $\mu(U k U$; $U g U, U h U)$ of pairs $(i, j)$ such that $U k=U g_{i} h_{j}$ is determined uniquely by $U g U, U h U$ and $U k U$. Then we define the product $U g U \cdot U h U$ by $\sum_{U k U} \mu(U k U ; U g U, U h U) U k U\left(=\sum_{i, j} U g_{i} h_{j}\right.$ formally). Let $\chi\left(r_{1}, \cdots, r_{n}\right)$ denote the matrix $\left(\begin{array}{ccc}p^{r_{1}} & & 0 \\ & \ddots & \\ 0 & & p^{r_{n}}\end{array}\right)$ where $r_{1}, \cdots, r_{n}$ are integers with $r_{1} \geq \cdots$ $\geq r_{n}$. Put

$$
\begin{aligned}
\pi_{0} & =U \chi(0, \cdots, 0) U \\
\pi_{1} & =U \chi(1,0, \cdots, 0) U \\
\pi_{2} & =U \chi(1,1,0, \cdots, 0) U \\
\vdots & \\
\pi_{n} & =U \chi(1, \cdots, 1) U,
\end{aligned}
$$

Received August 26, 1982. 
and $T(k)=\sum_{\substack{r_{1}+\cdots+r_{n}=k \\ r_{n} \geq 0}} U \chi\left(r_{1}, \cdots, r_{n}\right) U$ for $k \geq 0$ and $T(k)=0$ for $k<0$. Then we know, [10], the following

THEOREM. $H_{n}\left(\boldsymbol{Q}_{p}\right)$ is a commutative ring and

$$
\sum_{h=0}^{n}(-1)^{h} p^{h(h-1) / 2} T(k-h) \pi_{h}=0 \quad \text { for } k \geq 1 \text {. }
$$

Applying this, we give an induction formula of local densities.

Let $\tilde{S}$ be the set of non-singular symmetric $n \times n$ matrices over $\boldsymbol{Q}_{p}$. For $S_{1}, S_{2} \in \tilde{S}$ we define an equivalence relation $S_{1} \sim S_{2}$ by $S_{1}={ }^{t} T S_{2} T$ for some $T \in U$. By $\mathscr{L}(n)$ we denote the vector space over $\boldsymbol{Q}$ spanned by $[S], S \in \tilde{S}$, where [S] stands for the equivalence class containing $S$. Let $g$ be an element of $G$ and $U g U=U g_{1} \cup \ldots \cup U g_{s}$ a left cosets decomposition. Then we define the action of $U g U$ by

$$
[T] \mid U g U=p^{\left(\operatorname{ord}_{p}(\operatorname{det} g)\right)(n+1-m)} \sum_{i}\left[{ }^{t} g_{i}^{-1} T g_{i}^{-1}\right] \quad \text { for } T \in \tilde{S},
$$

where $m$ is a fixed integer. It is easy to see that this is well-defined.

Lemma 1. Let $g, h$ be elements of $G$. Then we have

$$
([T] \mid U g U)|U h U=[T]|(U g U \cdot U h U) \quad \text { for } T \in \tilde{S} \text {. }
$$

Proof. Let $U g U=U g_{1} \cup \cdots \cup U g_{s}, U h U=U h_{1} \cup \cdots \cup U h_{t}$. Then we have

$$
\begin{aligned}
([T] \mid U g U) \mid U h U & =p^{\left(\operatorname{ord}_{p}(\operatorname{det} g)\right)(n+1-m)} \sum_{i}\left[{ }^{t} g_{i}^{-1} T_{g_{i}^{-1}}\right] \mid U h U \\
& =p^{(\operatorname{ord} p(\operatorname{det} g h))(n+1-m)} \sum_{i, j}\left[{ }^{t} h_{j}^{-1} t g_{i}^{-1} T_{i^{-1}} h_{j}^{-1}\right] \\
& =[T]|(U h U \cdot U g U)=[T]|(U g U \cdot U h U) .
\end{aligned}
$$

Thus we get a homomorphism from $H_{n}\left(\boldsymbol{Q}_{p}\right)$ to $\operatorname{End}(\mathscr{L}(n))$ and the above theorem implies

Lemma 2. For $T \in \tilde{S}$ we have

$$
\sum_{h=0}^{n}(-1)^{h} p^{h(h-1) / 2}[T] \mid T(k-h) \pi_{h}=0 \quad \text { for } k \geq 1 .
$$

Let $S$ be a non-singular symmetric $m \times m$ matrix and even, that is, entries of $S$ are in $Z_{p}$ and diagonals are in $2 Z_{p}$. Suppose $m \geq n$. For $T \in \tilde{S} \cap M_{n}\left(Z_{p}\right)$ we put

$$
A_{p t}(T, S)=\#\left\{X \in M_{m, n}\left(Z_{p} /\left(p^{t}\right)\right) \mid{ }^{t} X S X \equiv T \bmod p^{t}\right\} .
$$


Then $2^{-\delta_{m, n}}\left(p^{t}\right)^{n(n+1) / 2-m n} A_{p t}(T, S)$ is independent of $t$ if $t$ is sufficiently large and we denote the value by $\alpha_{p}(T, S)\left(\delta_{m, n}\right.$ is the Kronecker's delta). Let $N=Z_{p}\left[u_{1}, \cdots, u_{n}\right], M=Z_{p}\left[v_{1}, \cdots, v_{m}\right]$ be lattices over $Z_{p}$ with $\mathrm{rk} N$ $=n, \operatorname{rk} M=m$ respectively. We define inner products $($,$) by \left(\left(u_{i}, u_{j}\right)\right)$ $=T,\left(\left(v_{i}, v_{j}\right)\right)=S$. We denote by $C_{p t}(T, S)$

$$
\sharp\left\{u: N \rightarrow M / p^{t} M^{\sharp} \mid \begin{array}{l}
(u x, u x) \equiv(x, x) \bmod 2 p^{t} Z_{p} \text { for } x \in N \text { and } u \\
\text { induces an injective mapping from } N / p N \text { to } M / p M
\end{array}\right\}
$$

and by $d_{p}(T, S) 2^{-\delta_{n, m}} p^{n \text { ord } p \operatorname{det} S} \times\left(p^{s}\right)^{n(n+1) / 2-m n} C_{p s}(T, S)$ where $s$ is any natural number such that $p^{s-1} S^{-1}$ is even and $M^{\#}$ is the dual lattice of $M$. Then the following is proved in [1].

Lemma 3. Keeping the above, we have

$$
\alpha_{p}(T, S)=2^{n \delta_{s, p}} \sum_{U \backslash U G U \ni g} p^{\left(\circ \mathrm{ord}_{p}(\operatorname{det} g)(n+1-m)\right.} d_{p}\left({ }^{(} g^{-1} T g^{-1}, S\right) .
$$

We define linear mappings $d, \alpha$ from $\mathscr{L}(n)$ to $Q$ by $d([T])=2^{n \tilde{\delta}_{2}, p} d_{p}(T, S)$, $\alpha([T])=\alpha_{p}(T, S)$. Then Lemma 3 means

$$
\alpha([T])=\sum_{U g U \in U G U} d([T] \mid U g U)=\sum_{k=0}^{\infty} d([T] \mid T(k))
$$

(for sufficiently large $k, d([T] \mid T(k))=0$ ).

THeOREM 1. Let $T^{(n)}, S^{(m)}(m \geq n)$ be even non-singular symmetric matrices. Then we have

$$
\left.2^{n \delta_{2}, p} d_{p}(T, S)=\sum_{h=0}^{n}(-1)^{h} p^{h(h-1) / 2+h(n+1-m)} \sum_{U \backslash \pi n h g} \alpha_{p}{ }^{t} g^{-1} T^{-1}, S\right) .
$$

Proof. Lemmas 2, 3 yield

$$
\begin{aligned}
\alpha\left(\sum_{h=0}^{n}(-1)^{h} p^{h(h-1) / 2}[T] \mid \pi_{h}\right) & =\sum_{h=0}^{n}(-1)^{h} p^{h(h-1) / 2} \sum_{i \geq 0} d\left([T] \mid \pi_{h} T(i-h)\right) \\
& =\sum_{i \geq 0} d\left(\sum_{h=0}^{n}(-1)^{h} p^{h(h-1) / 2}[T] \mid \pi_{h} T(i-h)\right) \\
& =d([T]) .
\end{aligned}
$$

Hereafter we assume that $p$ is an odd prime and $n=2$. Let $U\left(\begin{array}{ll}p & 0 \\ 0 & 1\end{array}\right) U=U g_{1} \cup \cdots \cup U g_{s}$ be a left cosets decomposition of $U\left(\begin{array}{ll}p & 0 \\ 0 & 1\end{array}\right) U$. Then $U\left(\begin{array}{ll}p & 0 \\ 0 & 1\end{array}\right) U=p g_{1}^{-1} U \cup \cdots \cup p g_{s}^{-1} U$ is a right cosets decomposition and we can take $\left\{g_{i}\right\}$ as $\left\{g_{i}^{-1}\right\}=\left\{\left(\begin{array}{cc}1 / p & 0 \\ 0 & 1\end{array}\right),\left(\begin{array}{ll}1 & a / p \\ 0 & 1 / p\end{array}\right) a \bmod p\right\}$. Put 


$$
T=\left(\begin{array}{cc}
\varepsilon_{1} p^{a_{1}} & 0 \\
0 & \varepsilon_{2} p^{a_{2}}
\end{array}\right), \quad \varepsilon_{i} \in Z_{p}^{\times}, \quad 0 \leq a_{1} \leq a_{2} .
$$

If $a \neq 0 \bmod p, a_{1}<a_{2}$, then

$$
T\left[\left(\begin{array}{cc}
1 & a / p \\
0 & 1 / p
\end{array}\right)\right] \sim\left(\begin{array}{cc}
\varepsilon_{1} p^{a_{1}-2} & 0 \\
0 & \varepsilon_{2} p^{a_{2}}
\end{array}\right) .
$$

If $a \neq 0 \bmod p, a^{2} \varepsilon_{1}+\varepsilon_{2} \neq 0 \bmod p$ and $a_{1}=a_{2}$, then

$$
T\left[\left(\begin{array}{cc}
1 & a / p \\
0 & 1 / p
\end{array}\right)\right] \sim\left(a^{2} \varepsilon_{1}+\varepsilon_{2}\right)\left(\begin{array}{cc}
p^{a_{1}-2} & 0 \\
0 & \varepsilon_{1} \varepsilon_{2} p^{a_{1}}
\end{array}\right) .
$$

If $a \neq 0 \bmod p, a^{2} \varepsilon_{1}+\varepsilon_{2} \equiv 0 \bmod p$ and $a_{1}=\alpha_{2}$, then

$$
T\left[\left(\begin{array}{ll}
1 & a / p \\
0 & 1 / p
\end{array}\right)\right] \sim\left(\begin{array}{cc}
p^{a_{1}-1} & 0 \\
0 & \varepsilon_{1} \varepsilon_{2} p^{a_{1}-1}
\end{array}\right) .
$$

Applying Theorem 1 and writing as $\alpha_{p}\left(\varepsilon_{1} p^{a_{1}}, \varepsilon_{2} p^{a_{2}}, S\right)$ instead of $\alpha_{p}(T, S)$, we have the following:

if $0 \leq a_{1}<a_{2}$, then

$$
\begin{aligned}
\alpha_{p}(T, S)= & d_{p}(T, S)+p^{4-m} \alpha_{p}\left(\varepsilon_{1} p^{a_{1}-2}, \varepsilon_{2} p^{a_{2}}, S\right)+p^{3-m} \alpha_{p}\left(\varepsilon_{1} p^{a_{1}}, \varepsilon_{2} p^{a_{2}-2}, S\right) \\
& -p^{7-2 m} \alpha_{p}\left(\varepsilon_{1} p^{a_{1}-2}, \varepsilon_{2} p^{a_{2}-2}, S\right) .
\end{aligned}
$$

if $0 \leq a_{1}=a_{2}$, then

$$
\begin{aligned}
\alpha_{p}(T, S)= & d_{p}(T, S)+p^{3-m} \alpha_{p}\left(\varepsilon_{1} p^{a_{1}-2}, \varepsilon_{2} p^{a_{1}}, S\right)+p^{3-m} \alpha_{p}\left(\varepsilon_{1} p^{a_{1}}, \varepsilon_{2} p^{a_{1}-2}, S\right) \\
& +p^{3-m} \sum_{\substack{a\left(a_{2}^{2} \varepsilon_{1}+\varepsilon_{2}\right) \neq 0(p)\\
}} \alpha_{p}\left(\left(a^{2} \varepsilon_{1}+\varepsilon_{2}\right) p^{a_{1}-2},\left(a^{2} \varepsilon_{1}+\varepsilon_{2}\right) \varepsilon_{1} \varepsilon_{2} p^{a_{1}}, S\right) \\
& +p^{3-m}\left(1+\chi\left(-\varepsilon_{1} \varepsilon_{2}\right)\right) \alpha_{p}\left(p^{a_{1}-1}, \varepsilon_{1} \varepsilon_{2} p^{a_{1}-1}, S\right) \\
& -p^{7-2 m} \alpha_{p}\left(\varepsilon_{1} p^{a_{1}-2}, \varepsilon_{2} p^{a_{1}-2}, S\right),
\end{aligned}
$$

where $\chi$ is the quadratic residue symbol defined modulo $p$.

TheOREM 2. Let $\varepsilon_{1}, \varepsilon_{2}$ be $p$-adic units in $Z_{p}$ and $0 \leq a_{1} \leq a_{2}\left(a_{i} \in Z\right)$ and $S$ a unimodular symmetric $m \times m$ matrix in $M_{m}\left(Z_{p}\right)$. Suppose that $m>2$ and $p$ is an odd prime. Let

$$
\chi(S)=\chi\left((-1)^{m / 2} \operatorname{det} S\right) \quad \text { or } \quad \chi\left((-1)^{(m-1) / 2} \operatorname{det} S\right)
$$

according to $2 \mid m$ or $2 \nmid m$ where $\chi$ is the quadratic residue symbol defined modulo $p$.

Put 


$$
\begin{aligned}
\beta_{0}(a, b)= & \left(1-\chi(S) p^{-m / 2}\right)\left(1-p^{2-m}\right)\left(1-p^{3-m}\right)^{-1} \\
& \times\left\{\sum_{k=0}^{a}\left(\chi(S) p^{2-m / 2}\right)^{k}-p^{(3-m) b / 2} \sum_{k=0}^{a}\left(\chi(S) p^{m / 2-1}\right)^{k}\right\} \\
\beta_{1}(a, b)= & p^{a / 2+(3-m) b / 2+1-m / 2}\left(1-p^{1-m}\right)\left(1-p^{(2-m)(a+1) / 2}\right)\left(1-p^{2-m}\right)^{-1}
\end{aligned}
$$

Then we have, for

$$
T=\left(\begin{array}{cc}
\varepsilon_{1} p^{a_{1}} & 0 \\
0 & \varepsilon_{2} p^{a_{2}}
\end{array}\right)
$$

(1) if $m \equiv 0 \bmod 2, a_{1}+a_{2} \equiv 0 \bmod 2$, then

$$
\begin{aligned}
\alpha_{p}(T, S)= & \beta_{0}\left(a_{1}, a_{1}+a_{2}\right)+p^{(3-m)\left(a_{1}+a_{2}\right) / 2}\left(1-\chi(S) p^{-m / 2}\right) \\
& \times\left(1+\chi(S) \chi\left(-\varepsilon_{1} \varepsilon_{2}\right) p^{1-m / 2}\right) \sum_{k=0}^{a_{1}}\left(\chi(S) p^{m / 2-1}\right)^{k},
\end{aligned}
$$

(2) if $m \equiv 0 \bmod 2, a_{1}+a_{2} \equiv 1 \bmod 2$, then

$$
\alpha_{p}(T, S)=\beta_{0}\left(a_{1}, a_{1}+a_{2}+1\right),
$$

(3) if $m \equiv 1 \bmod 2, a_{1} \equiv a_{2} \equiv 1 \bmod 2$, then

$$
\begin{aligned}
\alpha_{p}(T, S)= & \chi\left(-\varepsilon_{1} \varepsilon_{2}\right) \beta_{1}\left(a_{1}, a_{2}\right) \\
& +\left(1-p^{1-m}\right)\left(1-p^{(4-m)\left(a_{1}+1\right) / 2}\right)\left(1-p^{4-m}\right)^{-1},
\end{aligned}
$$

(4) if $m \equiv 1 \bmod 2, a_{1} \equiv 1, a_{2} \equiv 0 \bmod 2$, then

$$
\begin{aligned}
\alpha_{p}(T, S)= & \chi(S) \chi\left(\varepsilon_{2}\right) \beta_{1}\left(a_{1}, a_{2}\right) \\
& +\left(1-p^{1-m}\right)\left(1-p^{(4-m)\left(a_{1}+1\right) / 2}\right)\left(1-p^{4-m}\right)^{-1},
\end{aligned}
$$

(5) if $m \equiv 1 \bmod 2, a_{1} \equiv 0, a_{2} \equiv 1 \bmod 2$, then

$$
\begin{aligned}
\alpha_{p}(T, S)= & \chi(S) \chi\left(\varepsilon_{1}\right) \beta_{1}\left(a_{1}-1, a_{2}+1\right) \\
& +\left(1-p^{1-m}\right)\left(1-p^{(4-m) a_{1} / 2}\right)\left(1-p^{4-m}\right)^{-1} \\
& +p^{(4-m) a_{1} / 2}\left(1-p^{1-m}\right) \sum_{k=0}^{a_{2}-a_{1}}\left(\chi(S) \chi\left(\varepsilon_{1}\right) p^{(3-m) / 2}\right)^{k}
\end{aligned}
$$

(6) if $m \equiv 1 \bmod 2, a_{1} \equiv a_{2} \equiv 0 \bmod 2$, then

$$
\begin{aligned}
\alpha_{p}(T, S)= & p^{(3-m) / 2} \beta_{1}\left(a_{1}-1, a_{2}\right)+\left(1-p^{1-m}\right)\left(1-p^{(4-m) a_{1} / 2}\right)\left(1-p^{4-m}\right)^{-1} \\
& +p^{(4-m) a_{1} / 2}\left(1-p^{1-m}\right) \sum_{k=0}^{a_{2}-a_{1}}\left(\chi(S) \chi\left(\varepsilon_{1}\right) p^{(3-m) / 2}\right)^{k}
\end{aligned}
$$

Proof. First we note that if $N$ is a unimodular quadratic lattice and $L, M$ are quadratic lattices with integral scale, then $\alpha_{p}(N \perp L, N \perp M)$ $=\alpha_{p}(N, N \perp M) \alpha_{p}(L, M)$. If, hence, $a_{1}=0$, then 


$$
\alpha_{p}(T, S)=\alpha_{p}\left(\varepsilon_{1}, S\right) \alpha_{p}\left(\varepsilon_{2} p^{a_{2}}, S_{1}\right) \quad \text { where } S \sim\left(\begin{array}{cc}
\varepsilon_{1} & \\
& S_{1}
\end{array}\right),
$$

and the theorem follows from Hilfssatz 16 in [7]. It is easy to prove the theorem by the induction on $a_{1}+a_{2}$ in each case, using the induction formula before the theorem and $d_{p}(T, S)=\left(1-\chi(S) p^{-m / 2}\right)\left(1-p^{2-m}\right)$. $\left(1+\chi(S) p^{2-m / 2}\right)$ (resp. $\left.\left(1-p^{3-m}\right)\left(1-p^{1-m}\right)\right)$ for $2 \mid m$ (resp. $\left.2 \nmid m\right), 1 \leq a_{1} \leq a_{2}$.

Theorem 3. Let $S$ be a unimodular symmetric $m \times m$ matrix $(m \geq 3)$ and $p \neq 2$ put

$$
\alpha_{p}\left(\left(\begin{array}{cc}
\varepsilon_{1} p^{a_{1}} & \\
& \varepsilon_{2} p^{a_{2}}
\end{array}\right), S\right)=\alpha_{p}\left(\varepsilon_{1} p^{a_{1}}, \varepsilon_{2} p^{a_{2}}, S\right)
$$

Then we have

$$
\begin{aligned}
& \alpha_{p}\left(\varepsilon_{1} p^{a_{1}+2}, \varepsilon_{2} p^{a_{2}+2}, S\right)-p^{4-m} \alpha_{p}\left(\varepsilon_{1} p^{a_{1}}, \varepsilon_{2} p^{a_{2}}, S\right) \\
& =\alpha_{p}\left(\varepsilon_{1} p^{a_{1}}, \varepsilon_{2} p^{a_{2}+4}, S\right)-p^{4-m} \alpha_{p}\left(\varepsilon_{1} p^{a_{1}-2}, \varepsilon_{2} p^{a_{2}+2}, S\right) \\
& \text { for } 2 \leq a_{1} \leq a_{2}, \varepsilon_{i} \in Z_{p}^{\times} .
\end{aligned}
$$

If $m$ is even, then we have a detailed relation:

$$
\begin{aligned}
& \alpha_{p}\left(\varepsilon_{1} p^{a_{1}+1}, \varepsilon_{2} p^{a_{2}+1}, S\right)-\chi(S) p^{2-m / 2} \alpha_{p}\left(\varepsilon_{1} p^{a_{1}}, \varepsilon_{2} p^{a_{2}}, S\right) \\
& \quad=\alpha_{p}\left(\varepsilon_{1}, \varepsilon_{2} p^{a_{1}+a_{2}+2}, S\right) \quad \text { for } 0 \leq a_{1} \leq a_{2}, \varepsilon_{i} \in Z_{p}^{\times} .
\end{aligned}
$$

Proof. This follows immediately from Theorem 2.

Remark. By the Siegel formula relations in Theorem 3 imply relations among Fourier coefficients of Eisenstein series of degree 2. Especially detailed relations for even $m$ and $\chi(S)=1$ are equivalent to ResnikoffSaldaña-Maass relations. We note that $\alpha_{p}(\varepsilon T, S)=\alpha_{p}(T, S)\left(\varepsilon \in Z_{p}^{\times}\right)$if $S^{(m)}$ is unimodular and $m$ is even, because we have $S \sim \varepsilon S$ in this case.

Remark. We conjecture that the first relations in Theorem 3 hold for any regular symmetric matrix $S$ with $2+s \leqq a_{1} \leqq a_{2}$ where $s$ is the smallest integer such that $p^{s} S^{-1}$ is integral. This is the case for any binary matrix $S$, evaluating densities by reduction formulas. Note that if $n=m$, then $p^{-\left(\operatorname{ord}_{p}\left(|T||S|^{-1)}\right) / 2\right.} \alpha_{p}(T, S) / \alpha_{p}(S, S)$ is the number of quadratic lattices corresponding to $S$ which contain a fixed lattice corresponding to $T(\operatorname{rk} T=n, \operatorname{rk} S=m)$.

Theorem 1 is not so useful to evaluate local densities in general. At first the author got Theorem 2 in another way. (The formulas in [4] were not correct.) But at any rate Theorem 1 suggests that there are 
many relations among local densities since $d_{p}(T, S)$ is constant if $T \equiv$ $0 \bmod p^{s}$ such that $p^{s-1} S^{-1}$ is even.

We can evaluate local densities for $n=3$ by using Theorem 1 . But formulas are complicated. We give them for $n=3, m=3,4$.

Let $p$ be an odd prime and $S(\mathrm{rk} S=m$ ) a symmetric unimodular matrix. Put

$$
T\left(\varepsilon_{1} p^{a_{1}}, \varepsilon_{2} p^{a_{2}}, \varepsilon_{3} p^{a_{3}}\right)=\left(\begin{array}{lll}
\varepsilon_{1} p^{a_{1}} & & \\
& \varepsilon_{2} p^{a_{2}} & \\
& & \varepsilon_{3} p^{a_{3}}
\end{array}\right),
$$

where $\varepsilon_{i} \in Z_{p}^{\times}, 0 \leqq a_{1} \leqq a_{2} \leqq a_{3}$.

1) Case of $m=3$.

It is obvious that $\alpha_{p}\left(T\left(\varepsilon_{1} p^{a_{1}}, \varepsilon_{2} p^{a_{2}}, \varepsilon_{3} p^{a_{3}}\right), S\right)=0$ unless $\chi\left(\varepsilon_{1} \varepsilon_{2} \varepsilon_{3}|S|\right)=1$ and $a_{1}+a_{2}+a_{3} \equiv 0 \bmod 2$. Suppose that $\chi\left(\varepsilon_{1} \varepsilon_{2} \varepsilon_{3}|S|\right)=1$ and $a_{1}+a_{2}+a_{3}$ $\equiv 0 \bmod 2$. Then we have

$$
\begin{aligned}
& \alpha_{p}\left(T\left(\varepsilon_{1} p^{a_{1}}, \varepsilon_{2} p^{a_{2}}, \varepsilon_{3} p^{a_{3}}\right), S\right)=p^{\left(a_{1}+a_{2}+a_{3}\right) / 2} \cdot \alpha_{p}(S, S) \\
& \times \begin{cases}\left(1+\chi\left(-\varepsilon_{1} \varepsilon_{3}\right)\right) \sum_{0 \leqq k \leqq\left(a_{1}-1\right) / 2} p^{k} & \text { if } 2 \nmid a_{1}, 2 \mid a_{2}, \\
\left(1+\chi\left(-\varepsilon_{1} \varepsilon_{2}\right)\right) \sum_{0 \leqq k \leqq\left(a_{1}-1\right) / 2} p^{k} & \text { if } 2 \nmid a_{1}, 2 \nmid a_{2}, \\
\left(2+\chi\left(-\varepsilon_{2} \varepsilon_{3}\right)-\chi\left(-\varepsilon_{2} \varepsilon_{3}\right)^{a_{2}+1}\right) \sum_{0 \leqq k \leqq a_{1} / 2-1} p^{k} & \\
\quad+\sum_{0 \leqq k \leqq a_{2}-a_{1}} \chi\left(-\varepsilon_{2} \varepsilon_{3}\right)^{k} \cdot p^{a_{1} / 2} & \text { if } 2 \mid a_{1} .\end{cases}
\end{aligned}
$$

2) Case of $m=4$.

The value $\alpha_{p}\left(T\left(\varepsilon_{1} p^{a_{1}}, \varepsilon_{2} p^{a_{2}}, \varepsilon_{3} p^{a_{3}}\right), S\right)\left(1-\chi(S) p^{-2}\right)^{-1}\left(1-p^{-2}\right)^{-1}$ is equal to

$$
\begin{aligned}
& \left(\sum_{0 \leqq k \leqq a_{1}} \chi(S)^{k}\right)\left(\sum_{0 \leqq k \leqq a_{3}-a_{2}}\left(\chi\left(-\varepsilon_{1} \varepsilon_{2}\right) \chi(S)\right)^{k}\right) p^{\left(a_{1}+a_{2}\right) / 2} \\
& \quad+2\left(\sum_{0 \leqq k \leqq a_{1}} \chi(S)^{k}\right) \sum_{a_{1} \leqq j \leqq\left(a_{1}+a_{2}\right) / 2-1} p^{j} \\
& \quad+\sum_{0 \leqq j \leqq a_{1}-1}\left(\sum_{0 \leqq k \leqq j} \chi(S)^{k}\right) p^{j}\left\{\begin{array}{ll}
2 & \text { if } 2\left|a_{1}, 2\right| a_{2}, 2 \mid a_{3} \\
1+\chi(S) & \text { if } 2 \nmid a_{1}, 2 \nmid a_{2}, 2 \mid a_{3}
\end{array},\right.
\end{aligned}
$$

2.2) $\quad\left(1+\chi\left(-\varepsilon_{2} \varepsilon_{3}\right)\right)\left(\sum_{0 \leqq k \leqq a_{1}} \chi(S)^{k}\right) \sum_{a_{1}+1 \leqq j \leqq\left(a_{1}+a_{2}-1\right) / 2} p^{j}$

$$
+\sum_{0 \leqq j \leqq a_{1}}\left(\sum_{0 \leqq k \leqq j} \chi(S)^{k}\right) p^{j} \begin{cases}1+\chi\left(-\varepsilon_{2} \varepsilon_{3}\right) & \text { if } 2 \mid a_{1}, 2 \nmid a_{2}, 2 \nmid a_{3}, \\ 1+\chi\left(-\varepsilon_{2} \varepsilon_{3}\right) \chi(S) & \text { if } 2 \nmid a_{1}, 2\left|a_{2}, 2\right| a_{3},\end{cases}
$$

2.3) $\quad\left(\sum_{0 \leqq k \leqq a_{1}} \chi(S)^{k}\right)\left(\sum_{0 \leqq k \leqq a_{3}-a_{2}}\left(\chi\left(-\varepsilon_{1} \varepsilon_{2}\right) \chi(S)\right)^{k}\right) p^{\left(a_{1}+a_{2}\right) / 2}$

$$
+\left(1+\chi\left(-\varepsilon_{1} \varepsilon_{2}\right) \chi(S)\right)\left(\sum_{0 \leqq k \leqq a_{1}} \chi(S)^{k}\right) \sum_{a_{1} \leqq j \leqq\left(a_{1}+a_{2}\right) / 2-1} p^{j}
$$




$$
+\sum_{0 \leqq j \leqq a_{1}-1}\left(\sum_{0 \leqq k \leqq j} \chi(S)^{k}\right) p^{j} \begin{cases}1+\chi\left(-\varepsilon_{1} \varepsilon_{2}\right) \chi(S) & \text { if } 2\left|a_{1}, 2\right| a_{2}, 2 \nmid a_{3}, \\ 1+\chi\left(-\varepsilon_{1} \varepsilon_{2}\right) & \text { if } 2 \nmid a_{1}, 2 \nmid a_{2}, 2 \mid a_{3},\end{cases}
$$

2.4) $\quad\left(1+\chi\left(-\varepsilon_{1} \varepsilon_{3}\right) \chi(S)\right)\left(\sum_{0 \leqq k \leqq a_{1}} \chi(S)^{k}\right) \sum_{a_{1} \leqq j \leqq\left(a_{1}+a_{2}-1\right) / 2} p^{j}$

$$
+\sum_{0 \leqq j \leqq a_{1}-1}\left(\sum_{0 \leqq k \leqq j} \chi(S)^{k}\right) p^{j} \begin{cases}1+\chi\left(-\varepsilon_{1} \varepsilon_{3}\right) \chi(S) & \text { if } 2\left|a_{1}, 2 \nmid a_{2}, 2\right| a_{3}, \\ 1+\chi\left(-\varepsilon_{1} \varepsilon_{3}\right) & \text { if } 2 \nmid a_{1}, 2 \mid a_{2}, 2 \nmid a_{3} .\end{cases}
$$

Hence we see that

$$
\begin{aligned}
& \alpha_{p}\left(T\left(\varepsilon_{1} p^{a_{1}+2}, \varepsilon_{2} p^{a_{2}+2}, \varepsilon_{3} p^{a_{3}+2}\right), S\right)-p^{2} \alpha_{p}\left(T\left(\varepsilon_{1} p^{a_{1}}, \varepsilon_{2} p^{a_{2}}, \varepsilon_{3} p^{a_{3}}\right), S\right) \\
& \quad=\left\{\begin{array}{cl}
\alpha_{p}\left(T\left(\varepsilon_{1}, \varepsilon_{2} p^{a_{1}+a_{2}+4}, \varepsilon_{3} p^{a_{1}+a_{3}+4}\right), S\right) & \text { if } 2 \mid a_{1}, \\
+\chi(S) p \alpha_{p}\left(T\left(\varepsilon_{1}, \varepsilon_{2} p^{a_{1}+a_{2}+2}, \varepsilon_{3} p^{a_{1}+a_{3}+2}\right), S\right) & \text { if } 2 \nmid a_{1} . \\
\alpha_{p}\left(T\left(\varepsilon_{1} p, \varepsilon_{2} p^{a_{1}+a_{2}+3}, \varepsilon_{3} p^{a_{1}+a_{3}+3}\right), S\right) &
\end{array}\right.
\end{aligned}
$$

\section{REFERENCES}

[1] Y. Kitaoka, Modular forms of degree $n$ and representation by quadratic forms II, Nagoya Math. J., 87 (1982), 127-146.

[2] H. Maass, Die Fourier koeffizienten der Eisensteinreihen zweiten Grades, Mat.Fys. Medd. Danske Vid. Selsk., 34, Nr. 7 (1964).

[3] — U Über die Fourier koeffizienten der Eisensteinreihen zweiten Grades, ibid. 38, Nr. 14 (1972).

[4] M. Ozeki, On the evaluation of certain generalized Gauss sums in non-dyadic case, preprint, (1981).

[ 5 ] - On certain generalized Gaussian sums, Proc. Japan Acad., 58 (1982), 223226.

[ 6 ] H. L. Resnikoff, R. L. Saldaña, Some properties of Fourier coefficients of Eisenstein series of degree two, J. reine angew. Math., 265 (1974), 90-109.

[ 7 ] C. L. Siegel, Über die analytische Theorie der quadratischen Formen, Ann. of Math., 36 (1935), 527-606.

[ 8 ] — Einführung in die Theorie der Modulfunktionen n-ten Grades, Math. Ann., 116 (1939), 617-657.

[9] — Über die Fourierschen Koeffizienten der Eisensteinschen Reihen, Mat.-Fys. Medd. Danske Vid. Selsk. 34, Nr. 6 (1964).

[10] T. Tamagawa, On the $\zeta$-functions of a division algebra, Ann. of Math., 77 (1963), $387-405$.

Department of Mathematics

Faculty of Science

Nagoya University

Chikusa-ku, Nagoya 464

Japan 